\title{
Bayesian Premium Estimators for Mixture of Two Gamma Distributions Under Squared Error, Entropy and Linex Loss Functions: With Informative and Non Informative Priors
}

\author{
Fatma Zohra Attoui ${ }^{1}$, Halim Zeghdoudi ${ }^{1 \star \star}$, and Ahmed Saadoun ${ }^{1}$ \\ ${ }^{1}$ LaPS Laboratory, Badji-Mokhtar University, Box 12, Annaba, 23000, Algeria
}

\section{ARTICLE INFO}

Article History

Received Jan 22018

Accepted April 12018

Keywords

Zeghdoudi distribution

gamma distribution

loss function

Bayesian premium.

2000 Mathematics Subject Classification

$62 \mathrm{P} 05,62.1$

\begin{abstract}
In this paper, we consider the Zeghdoudi distribution as the conditional distribution of $X_{n} \mid \theta$, we focus on estimation of the Bayesian premium under three loss functions (squared error which is symmetric, Linex and entropy, which are asymmetric), using non-informative and informative priors (the extension of Jeffreys and Gamma priors) respectively. Because of its difficulty and non linearity, we use a numerical approximation for computing the Bayesian premium.
\end{abstract}

(c) 2018 The Authors. Published by Atlantis Press SARL. This is an open access article under the CC BY-NC license (http://creativecommons.org/licenses/by-nc/4.0/).

\section{INTRODUCTION}

Credibility theory is a rating technique in actuarial science which can be seen as one of the quantitative tools that allow the insurers to perform experience rating, that is, to adjust future premiums based on past experiences. We focused on a popular tool in credibility theory which is the Bayesian premium estimator developed by [1], considering the Zeghdoudi distribution (ZD) as a claim distribution.

The ZD [2] has been overlooked in the literature from 2017, the idea is based on mixtures of the ordinary exponential $(\theta)$ and Gamma $(3, \theta)$ distributions, is one of the distributions of waiting time, life testing, and reliability theory and some classical statistics properties are investigated by [3]. There are a lot of papers is based on mixtures of the distribution, such as distribution Introduced by Lindley in 1958. Lindley distribution originally developed by Lindley [4], Zeghdoudi and Nedjar [5-8], Zeghdoudi and Lazri [9] introduced a new distribution, named gamma Lindley, pseudo Lindley, Lindley-Pareto and others like Shanker et al. [10,11] and Ghitany et al. [3] and Sankaran [12] and Ghitany and Al-Mutairi [13].

Recently Krishna and Kumar [14] use the maximum likelihood and Bayesian approach, however they did not consider it for the complete data set using various loss functions, a study of the effect of some loss functions on Bayes Estimate and posterior risk for the Lindley distribution is made by Sajid Ali et al. [15]. Metiri et al. [16] explain the derivation of posterior distributions for the Lindley distribution under Linex loss functions using informative and non-informative priors.

Let $x_{1}, x_{2}, \ldots, x_{n}$ be independent and identically distributed lifetimes from a ZD with an unknown parameter $\theta$. The probability density function is given by:

$$
\left\{f_{Z D}(x, \theta)=\begin{array}{cl}
\frac{\theta^{3} x(1+x) e^{-\theta x}}{\theta+2}, & x, \theta>0 \\
0, & \text { otherwise. }
\end{array}\right.
$$


The likelihood function for a random sample $x_{1}, x_{2} \ldots, x_{n}$ which is taken from $\mathrm{ZD}$ is:

$$
L(x, \theta)=\left(\frac{\theta^{3}}{(\theta+2)}\right)^{n} \Pi_{i=1}^{n}\left(x_{i}^{2}+x_{i}\right) e^{-\theta \sum_{i=1}^{n} x_{i}}, x, \theta>0
$$

The article is organized as follows, Section 2 explains the we derive this estimator under entropy loss which is asymmetric and squared error loss and Linex loss which is a symmetric loss function with informative and non-informative priors. The paper finds a solution to this problem by deriving this estimator using numerical approximation (Lindley approximation) which is one of the suitable approximation methods for solving such problems, it approaches the ratio of the integrals as a whole and produces a single numerical result. In Section 3, Simulation study using Monte Carlo method is then performed to evaluate this estimator and mean squared error technique is made to compare the Bayesian premium estimator under the above loss functions.

\section{DERIVATION OF BAYESIAN PREMIUMS}

To obtain Bayesian premium estimators, we assume that $\theta$ is a real valued random variable with probability density function $\pi(\theta)$. The posterior distribution of $\theta$, i.e., recall that the conditional distribution of $X_{n} \mid \theta$ is the ZD and the distribution of $\Theta$ is assumed to be known in the present section.

$f(\theta \mid x)$ is the posterior distribution of $\theta$ given the data. In this section we consider estimation of the Bayesian premium $\mathbf{P}^{B}$ based on the above mentioned priors and loss functions.

\subsection{Bayesian Premium Estimators Under Squared Error Loss Function}

The squared error loss function was proposed by [17] and [4] to develop least squares theory. It is defined as

$$
L(\hat{\theta}, \theta)=(\hat{\theta}-\theta)^{2} \text {. }
$$

In the actuarial literature, we write

$$
L\left(\mathbf{P}_{\mathrm{SELF}}^{B}, \mu(\theta)\right)=\left(\mathbf{P}_{\mathrm{SELF}}^{B}-\mu(\theta)\right)^{2}
$$

The Bayesian premium $\mathbf{P}_{\text {SELF }}^{B}$ is the estimator of $\mu(\theta)$, it is to be chosen such that the posterior expectation of the squared error loss function

$$
E[L(\hat{\theta}, \theta)]=\int_{0}^{\infty} L(\hat{\theta}, \theta) f(\theta \mid x) d \theta=\int_{0}^{\infty}\left(\mathbf{P}_{\mathrm{SELF}}^{B}-\mu(\theta)\right) f(\theta \mid x) d \theta,
$$

is minimum.

$$
\mathbf{P}_{\mathrm{SELF}}^{B}=E[\mu(\Theta) \mid x]=\int_{0}^{\infty} \mu(\theta) f(\theta \mid x) d \theta
$$

where

$$
\mu(\theta)=E[x \mid \Theta]=\frac{2(\theta+3)}{\theta(\theta+2)}
$$

is the individual premium.

\subsubsection{Posterior distribution using the extension of Jeffreys prior}

Bayesian approach makes use of ones prior knowledge about the parameters as well as the available data. When ones prior knowledge about the parameter is not available, it is possible to make use of the non-informative prior in Bayesian analysis.

Since we have no knowledge on the parameters, we seek to use the extension of Jeffreys' prior information, where Jeffreys' prior is the square root of the determinant of the Fisher information. We find Jeffrey prior by taking $\pi(\theta)=\sqrt{I(\theta)}$, where

$$
I(\theta)=-E\left[\frac{\partial^{2} \log f(x ; \theta)}{\partial \theta^{2}}\right]=\frac{2\left(\theta^{2}+6 \theta+6\right)}{\theta^{2}(\theta+2)^{2}},
$$


The extension of Jeffreys distribution is assumed as non-informative prior for the parameter $\theta$. It was proposed by [18] and [19], it is given as:

$$
\begin{gathered}
I(\theta)=\frac{2\left(\theta^{2}+6 \theta+6\right)}{\theta^{2}(\theta+2)^{2}}, \\
\pi(\theta)=[I(\theta)]^{c}=k\left[\frac{2\left(\theta^{2}+6 \theta+6\right)}{\theta^{2}(\theta+2)^{2}}\right]^{c}, \theta, c>0, k \text { is a constant. }
\end{gathered}
$$

Combining Eq. (6) with the likelihood function of $\mathrm{ZD}$, the posterior distribution of parameter $\theta$ given the data $\left(x_{1}, x_{2} \ldots, x_{n}\right)$ is derived as follows:

$$
f(\theta \mid x)=\frac{\Pi_{i=1}^{n} L\left(x_{i}, \theta\right) \pi(\theta)}{\int_{0}^{\infty} \Pi_{i=1}^{n} L\left(x_{i}, \theta\right) \pi(\theta) d \theta}=\frac{\frac{\theta^{(3 n-2 c)}}{(\theta+2)^{n+2 c}}\left(\theta^{2}+6 \theta+6\right)^{c} e^{-\theta \sum_{i=1}^{n} x_{i}}}{\int_{0}^{\infty} \frac{\theta^{(3 n-2 c)}}{(\theta+2)^{n+2 c}}\left(\theta^{2}+6 \theta+6\right)^{c} e^{-\theta \sum_{i=1}^{n} x_{i}} d \theta}, \theta>0 .
$$

According to the squared error loss function, the corresponding Bayesian premium estimator is derived by substituting the posterior distribution Eq. (6) in Eq. (3), as follows:

$$
\begin{gathered}
\mathbf{P}_{\mathrm{SELF}}^{B}=\int_{0}^{\infty} \mu(\theta) f(\theta \mid x) d \theta \\
\mathbf{P}_{\mathrm{SELF}}^{B}=\frac{\int_{0}^{\infty} \frac{2 \theta^{(3 n-2 c-1)}}{(\theta+2)^{n+2 c+1}}(\theta+3)\left(\theta^{2}+6 \theta+6\right)^{c} e^{-\theta \sum_{i=1}^{n} x_{i}} d \theta}{\int_{0}^{\infty} \frac{\theta^{(3 n-2 c)}}{(\theta+2)^{n+2 c}}\left(\theta^{2}+6 \theta+6\right)^{c} e^{-\theta \sum_{i=1}^{n} x_{i}} d \theta}, \theta>0
\end{gathered}
$$

We know that only combinations of unidimensional exponential family members with their natural conjugate priors yield linear Bayesian premiums (exact credibility formula).

The natural conjugate priors which give us a credibility premium formula are Gamma, Beta, and normal density. Since, Poisson, exponential, geometric, binomial and normal distribution belong to the exponential family of distributions.

It may be noted here that the posterior distribution $f(\theta \mid x)$ takes a ratio form that it gives not a credibility formula and involves an integration in the denominator and cannot be reduced to a closed form. Hence, the evaluation of the posterior expectation of obtaining the Bayesian premium of $\theta$ will be tedious. Among the various methods suggested to approximate the ratio of the integrals of the above form, perhaps the simplest one is Lindley's [19] approximation method, which approaches the ratio of the integrals as a whole and produces a single numerical result. Thus, we propose the use of Lindley's [19] approximation for obtaining the Bayesian premium of $\theta$. Many authors have used this approximation for obtaining the Bayes estimators for some distributions; see among others, [20] and [21].

If $n$ is sufficiently large, according to Lindley [19], any ratio of the integral of the form

$$
I(x)=E[h(\Theta)]=\frac{\int_{\theta} h(\theta) \exp [L(\theta, x)+g(\theta)] d \theta}{\int_{\theta} \exp [L(\theta, x)+g(\theta)] d \theta}, \theta>0
$$

where $h(\theta)$, function of $\theta$ only;

$$
\begin{aligned}
& L(\theta, x), \log \text { of likelihood; } \\
& g(\theta), \log \text { of prior of } \theta .
\end{aligned}
$$

Thus,

$$
\begin{gathered}
I(x)=h(\hat{\theta})+0.5\left[\left(\hat{h}_{\theta \theta}+2 \hat{h}_{\theta} \hat{p}_{\theta}\right) \hat{\sigma}_{\theta \theta}\right]+0.5\left[\left(\hat{h}_{\theta} \hat{\sigma}_{\theta \theta}\right)\left(\hat{L}_{\theta \theta \theta} \hat{\sigma}_{\theta \theta}\right)\right], \\
\text { where } \hat{h}_{\theta}=\frac{\partial h(\hat{\theta})}{\partial \hat{\theta}}, \hat{h}_{\theta \theta}=\frac{\partial^{2} h(\hat{\theta})}{\partial \hat{\theta}^{2}}, \hat{p}_{\theta}=\frac{\partial g(\hat{\theta})}{\partial \hat{\theta}}, \hat{L}_{\theta \theta}=\frac{\partial^{2} L(\hat{\theta})}{\partial \hat{\theta}^{2}}, \hat{\sigma}_{\theta \theta}=-\frac{1}{\hat{L}_{\theta \theta}}, \hat{L}_{\theta \theta \theta}=\frac{\partial^{3} L(\hat{\theta})}{\partial \hat{\theta}^{3}} .
\end{gathered}
$$

After substituting the value of $f(\theta \mid x)$, it may be written as:

$$
\mathbf{P}_{\mathrm{SELF}}^{B}=E[\mu(\Theta) \mid x]=\frac{\int_{\theta} \mu(\theta) \exp [L(\theta, x)+g(\theta)] d \theta}{\int_{\theta} \exp [L(\theta, x)+g(\theta)] d \theta}, \theta>0,
$$


where

$$
\begin{gathered}
h(\theta)=\mu(\theta)=\frac{2(\theta+3)}{\theta(\theta+2)}, \\
L(\theta, x)=3 n \ln \theta-n \ln (\theta+2)+\sum_{i=1}^{n} \ln \left(x_{i}+x_{i}^{2}\right)-\theta \sum_{i=1}^{n} x_{i}, \\
g(\theta)=c\left[\ln \left(2 \theta^{2}+12 \theta+12\right)-2 \ln \left(\theta^{2}+2 \theta\right)\right] .
\end{gathered}
$$

It may easily be verified that

$$
\begin{aligned}
& \hat{h}_{\theta}=-\frac{2\left(\theta^{2}+6 \theta+6\right)}{\left(\theta^{2}+2 \theta\right)^{2}}, \hat{h}_{\theta \theta}=\frac{4\left(\theta^{3}+9 \theta^{2}+18 \theta+12\right)}{\left(\theta^{2}+2 \theta\right)^{3}}, \hat{p}_{\theta}=2 c\left(\frac{\theta+3}{\theta^{2}+6 \theta+6}-\frac{2 \theta+2}{\theta^{2}+2 \theta}\right), \\
& \hat{L}_{\theta \theta}=-\frac{3 n}{\theta^{2}}+\frac{n}{(\theta+2)^{2}}, \quad \hat{\sigma}_{\theta \theta}=\frac{\theta^{2}(\theta+2)^{2}}{n\left[3(\theta+2)^{2}-\theta^{2}\right]}, \quad \hat{L}_{\theta \theta \theta}=\frac{6 n}{\theta^{3}}-\frac{2 n}{(\theta+2)^{3}} .
\end{aligned}
$$

Then, we get

$$
\mathbf{P}_{\mathrm{SELF}}^{B}=E[\mu(\Theta) \mid x]
$$

$$
\begin{aligned}
\mathbf{P}_{\mathrm{SELF}}^{B}= & \frac{2\left(\hat{\theta}^{2}+3\right)}{\hat{\theta}(\hat{\theta}+2)}+0.5\left[\left(\left(\frac{2 \hat{\theta}^{6}+60 \hat{\theta}^{4}+72 \hat{\theta}^{2}+48}{\left(\hat{\theta}^{3}+2 \hat{\theta}\right)^{3}}\right)-4 c\left(\frac{2 \hat{\theta}^{3}+16 \hat{\theta}}{\hat{\theta}^{4}+16 \hat{\theta}+12}-\frac{3 \hat{\theta}^{2}+2}{\hat{\theta}^{3}+2 \theta}\right)\left(\frac{\hat{\theta}^{4}+16 \hat{\theta}^{2}+12}{\left(\hat{\theta}^{3}+2 \hat{\theta}\right)^{2}}\right)\right)\right. \\
& \left.\frac{\hat{\theta}^{2}\left(\hat{\theta}^{2}+2\right)}{n\left[3\left(\hat{\theta}^{2}+2\right)^{2}+2 \hat{\theta}^{2}\left(2-\hat{\theta}^{2}\right)\right]}\right]-0.5\left[\left[\frac{\left.\hat{\theta}^{2} \hat{\theta}^{2}+2\right)}{n\left[3\left(\hat{\theta}^{2}+2\right)^{2}+\hat{\theta}^{2}\left(2-\hat{\theta}^{2}\right)\right]}\right]^{2}\left(\frac{6 n}{\hat{\theta}^{3}}-\frac{4 n\left(\hat{\theta}^{3}-6 \hat{\theta}\right)}{\left(\hat{\theta}^{2}+2\right)^{3}}\right)\left(\frac{\hat{\theta}^{4}+16 \hat{\theta}^{2}+12}{\left(\hat{\theta}^{3}+2 \hat{\theta}\right)^{2}}\right)\right] .
\end{aligned}
$$

\subsubsection{Posterior distribution using the inverted gamma prior}

The inverted gamma (IG) prior is a good life distribution model which represents the reciprocal of a variable distributed according to the gamma distribution. It is observed that if $\theta$ has an IG $(\alpha ; \beta)$ distribution then $\frac{1}{\theta}$ has a gamma $(\alpha ; \beta)$ distribution.

It is given as

$$
\pi(\theta)=\frac{\beta^{\alpha}}{\Gamma(\alpha)} \frac{1}{\theta^{\alpha+1}} e^{-\frac{\beta}{\theta}} ; \alpha, \beta, \theta>0 .
$$

The first two moments of $I G(\alpha, \beta)$ are

$$
E(\Theta)=\frac{\beta}{\alpha-1}, \text { and } \operatorname{Var}(\theta)=\frac{\beta^{2}}{(\alpha-1)^{2}(\alpha-2)} .
$$

Now, using the likelihood of ZD and the IG prior, the posterior distribution for the parameter $\theta$ given the data $\left(x_{1}, x_{2} \ldots, x_{n}\right)$ takes the form

$$
\begin{gathered}
f(\theta \mid x)=\frac{\Pi_{i=1}^{n} L\left(x_{i} \mid \theta\right) \pi(\theta)}{\int_{0}^{\infty} \Pi_{i=1}^{n} L\left(x_{i} \mid \theta\right) \pi(\theta) d \theta} \\
f(\theta \mid x)=\frac{\frac{\theta^{3 n-(\alpha+1)}}{(2+\theta)^{n}} e^{-\frac{\beta}{\theta}-\theta \sum_{i=1}^{n} x_{i}}}{\int_{0}^{\infty} \frac{\theta^{3 n-(\alpha+1)}}{(2+\theta)^{n}} e^{-\frac{\beta}{\theta}-\theta \sum_{i=1}^{n} x_{i}} d \theta} .
\end{gathered}
$$

Now, according to the squared error loss function, the corresponding Bayes' estimator for the parameter $\theta$ is derived by substituting the posterior distribution Eq. (16) in Eq. (17), as follows:

$$
\mathbf{P}_{\mathrm{SELF}}^{B}=\frac{\int_{0}^{\infty} \frac{\theta^{3 n-(\alpha+2)}}{(2+\theta)^{n+1}}(\theta+3) e^{-\frac{\beta}{\theta}-\theta \sum_{i=1}^{n} x_{i}} d \theta}{\int_{0}^{\infty} \frac{\theta^{3 n-(\alpha+1)}}{(2+\theta)^{n}} e^{-\frac{\beta}{\theta}-\theta \sum_{i=1}^{n} x_{i}} d \theta}, \theta>0 .
$$


Following the procedure as discussed above, we have

$$
g(\theta)=\beta \ln \alpha-\ln \Gamma(\beta)-(\alpha+1) \ln \theta-\frac{\beta}{\theta} .
$$

It may easily be verified that

$$
\begin{aligned}
& \hat{h}_{\theta}=-\frac{2\left(\theta^{2}+6 \theta+6\right)}{\left(\theta^{2}+2 \theta\right)^{2}}, \hat{h}_{\theta \theta}=\frac{4\left(\theta^{3}+9 \theta^{2}+18 \theta+12\right)}{\left(\theta^{2}+2 \theta\right)^{3}}, \hat{p}_{\theta}=\frac{\beta}{\theta^{2}}-\frac{\alpha+1}{\theta}, \\
& \hat{L}_{\theta \theta}=-\frac{3 n}{\theta^{2}}+\frac{n}{(\theta+2)^{2}}, \quad \hat{\sigma}_{\theta \theta}=\frac{\theta^{2}(\theta+2)^{2}}{n\left[3(\theta+2)^{2}-\theta^{2}\right]}, \quad \hat{L}_{\theta \theta \theta}=\frac{6 n}{\theta^{3}}-\frac{2 n}{(\theta+2)^{3}} .
\end{aligned}
$$

We get after simplification

$$
\begin{aligned}
\left.\mathbf{P}_{\mathrm{SELF}}^{B}=E[\mu(\Theta) \mid x]=\frac{2(\hat{\theta}+3)}{\hat{\theta}(\hat{\theta}+2)}+0.5\left[\begin{array}{c}
\left(\frac{2 \hat{\theta}^{6}+60 \hat{\theta}^{4}+72 \hat{\theta}^{2}+48}{\left(\hat{\theta}^{3}+2 \hat{\theta}\right)^{3}}\right)- \\
2\left(\frac{\beta}{\theta^{2}}-\frac{\alpha+1}{\theta}\right)\left(\frac{\hat{\theta}^{4}+16 \hat{\theta}^{2}+12}{\left(\hat{\theta}^{3}+2 \hat{\theta}\right)^{2}}\right)
\end{array}\right) \frac{\hat{\theta}^{2}\left(\hat{\theta}^{2}+2\right)}{n\left[3\left(\hat{\theta}^{2}+2\right)^{2}+2 \hat{\theta}^{2}\left(2-\hat{\theta}^{2}\right)\right]}\right] \\
-0.5\left[\frac{\hat{\theta}^{2}\left(\hat{\theta}^{2}+2\right)}{n\left(3\left(\hat{\theta}^{2}+2\right)^{2}+2 \hat{\theta}^{2}\left(2-\hat{\theta}^{2}\right)\right)}\right]^{2}\left(\frac{6 n}{\left.\left.\hat{\theta}^{3}-\frac{4 n\left(\hat{\theta}^{3}-6 \hat{\theta}\right)}{\left(\hat{\theta}^{2}+2\right)^{3}}\right)\left(\frac{\hat{\theta}^{4}+16 \hat{\theta}^{2}+12}{\left(\hat{\theta}^{3}+2 \hat{\theta}\right)^{2}}\right)\right] .}\right.
\end{aligned}
$$

\subsection{Bayesian Premium Estimators Under Linex Loss Function}

The linex (linear-exponential) loss function (the name linex is justified by the fact that this asymmetric loss function rises approximately linearly on one side of zero and approximately exponentially on the other side) which is asymmetric, was introduced by [25-26]. It may be expressed as:

$$
L(\hat{\theta}, \theta)=\exp (a(\hat{\theta}-\theta))-a(\hat{\theta}-\theta)-1, a \neq 0
$$

The sign and magnitude of the shape parameter $a$ reflects the direction and degree of asymmetry, respectively. (If $a>0$, the over estimation is more serious than underestimation, and vice-versa). For $a$ closed to zero, the Linex loss is approximately squared error loss and therefore almost symmetric.

The posterior expectation of the linex loss function equation is:

$$
E[L(\hat{\theta}, \theta)] \propto \exp (a \hat{\theta}) E[\exp (-a \theta)]-a(\hat{\theta}-E(\theta))-1
$$

By result of [5], the estimator of $\theta$ under the linex loss $\hat{\theta}$ which minimizes the above equation is given by

$$
\hat{\theta}=-\frac{1}{a} \ln \left[E\left[e^{-a \Theta}\right]\right]
$$

In our study, the aim is to find the Bayesian premium estimator $\mathbf{P}_{\mathrm{LIN}}^{B}$ which is the value that minimizes the above equation, it is given by:

$$
\mathbf{P}_{\mathrm{LIN}}^{B}=-\frac{1}{a} \ln \left[E\left[e^{-a \mu(\Theta)} \mid x\right]\right]
$$

When the expectation $E\left[e^{-a \mu(\Theta)}\right]$ exists and finite [27].

Thomson and Basu in [25] identified a family of loss functions $L(\Delta)$ where $\Delta$ is either the estimation error $(\hat{\theta}-\theta)$, such that

- $L(0)=0$;

- $L(\Delta)>L(-\Delta)>0$ for all $\Delta>0$ 
- $L(\Delta)$ is twice differentiable with $L^{\prime}(0)=0$ and $L^{\prime \prime}(\Delta)>0$ for all $\Delta \neq 0$;

- $L^{\prime}(\Delta)>-L^{\prime}(-\Delta)>0$ for all $\Delta>0$.

\subsubsection{Posterior distribution using the extension of Jeffreys prior}

Using the linex loss function, the corresponding Bayes estimator of the parameter $\theta$ is as follows:

$$
\begin{aligned}
\mathbf{P}_{\mathrm{LIN}}^{B}=-\frac{1}{a} \ln E\left[e^{-a \mu(\Theta)} \mid x\right] . \\
E\left[e^{-a \mu(\Theta)} \mid x\right]=\int_{0}^{\infty} e^{-a \mu(\theta)} f(\theta \mid x) d \theta \\
=\frac{\int_{0}^{\infty} \frac{\theta^{(3 n-2 c)}}{(\theta+2)^{n+2 c}}\left(\theta^{2}+6 \theta+6\right)^{c} e^{-\left(\theta \sum_{i=1}^{n} x_{i}+a \mu(\theta)\right)} d \theta}{\int_{0}^{\infty} \frac{\theta^{(3 n-2 c)}}{(\theta+2)^{n+2 c}}\left(\theta^{2}+6 \theta+6\right)^{c} e^{-\theta \sum_{i=1}^{n} x_{i}} d \theta} \\
=\frac{\int_{\theta} h(\theta) \exp [L(\theta, x)+g(\theta)] d \theta}{\int_{\theta} \exp [L(\theta, x)+g(\theta)] d \theta}, \theta>0
\end{aligned}
$$

Following the same steps explained above, we have

$$
h(\theta)=e^{-a \mu(\theta)}
$$

$L(\theta, x)$ and $g(\theta)$ are the same as those given in Eqs. (12) and (13).

$$
\begin{gathered}
\hat{h}_{\theta}=-a \mu^{\prime}(\theta) e^{-a \mu(\theta)}, \hat{h}_{\theta \theta}=-a\left(\mu^{\prime \prime}(\theta)-a \mu^{\prime 2}(\theta)\right) e^{-a \mu(\theta)}, \hat{p}_{\theta}=2 c\left(\frac{\theta+3}{\theta^{2}+6 \theta+6}-\frac{2 \theta+2}{\theta^{2}+2 \theta}\right), \\
\hat{L}_{\theta \theta}=-\frac{3 n}{\theta^{2}}+\frac{n}{(\theta+2)^{2}}, \quad \hat{\sigma}_{\theta \theta}=\frac{\theta^{2}(\theta+2)^{2}}{n\left[3(\theta+2)^{2}+\theta^{2}\right]}, \quad \hat{L}_{\theta \theta \theta}=\frac{6 n}{\theta^{3}}-\frac{2 n}{(\theta+2)^{3}},
\end{gathered}
$$

where

$$
\mu^{\prime}(\theta)=\frac{\partial \mu(\theta)}{\partial \theta}=-\left(\frac{2\left(\theta^{2}+6 \theta+6\right)}{\left(\theta^{2}+2 \theta\right)^{2}}\right), \mu^{\prime \prime}(\theta)=\frac{\partial^{2} \mu(\theta)}{\partial \theta^{2}}=\left(\frac{4\left(\theta^{3}+9 \theta^{2}+18 \theta+12\right)}{\left(\theta^{2}+2 \theta\right)^{3}}\right)
$$

Then, we get

$$
\mathbf{P}_{\mathrm{LIN}}^{B}=-\frac{1}{\alpha} \ln \left[\begin{array}{c}
\left(-\alpha\left(\mu^{\prime \prime}(\hat{\theta})-a\left(\mu^{\prime}(\hat{\theta})\right)^{2}\right) e^{-a \mu(\hat{\theta})}\right) \\
e^{-\alpha \mu(\hat{\theta})}+0.5\left(-4\left(-a \mu^{\prime}(\hat{\theta}) e^{-a \mu(\hat{\theta})}\right)\left(c\left(\frac{2 \theta^{3}+16 \theta}{\theta^{4}+16 \theta+12}-\frac{3 \theta^{2}+2}{\theta^{3}+2 \theta}\right)\right) \frac{\hat{\theta}^{2}\left(\hat{\theta}^{2}+2\right)}{n\left[3\left(\hat{\theta}^{2}+2\right)^{2}+2 \hat{\theta}^{2}\left(2-\hat{\theta}^{2}\right)\right]}\right. \\
+0.5\left(\left(\frac{\hat{\theta}^{2}\left(\hat{\theta}^{2}+2\right)}{n\left[3\left(\hat{\theta}^{2}+2\right)^{2}+2 \hat{\theta}^{2}\left(2-\hat{\theta}^{2}\right)\right]}\right)^{2}\left(-a \mu^{\prime}(\hat{\theta}) e^{-a \mu(\hat{\theta})}\right)\left(\frac{6 n}{\hat{\theta}^{3}}-\frac{4 n\left(\hat{\theta}^{3}-6 \hat{\theta}\right)}{\left.\left(\hat{\theta}^{2}+2\right)^{3}\right)}\right)\right.
\end{array}\right]
$$

\subsubsection{Posterior distribution using the IG prior}

The corresponding Bayesian premium estimator under the linex loss function is:

$$
\mathbf{P}_{\mathrm{LIN}}^{B}=-\frac{1}{a} \ln E\left[e^{-a \mu(\Theta)} \mid x\right]
$$




$$
\begin{aligned}
E\left[e^{-a \mu(\Theta)} \mid x\right] & =\int_{0}^{\infty} e^{-a \mu(\theta)} f(\theta \mid x) d \theta \\
& =\frac{\int_{0}^{\infty} \frac{\theta^{3 n-(\alpha+1)}}{(2+\theta)^{n}} e^{-\left(\frac{\beta}{\theta}+\theta \sum_{i=1}^{n} x_{i}+a \mu(\theta)\right)} d \theta}{\int_{0}^{\infty} \frac{\theta^{3 n-(\alpha+1)}}{(2+\theta)^{n}} e^{-\left(\frac{\beta}{\theta}+\theta \sum_{i=1}^{n} x_{i}\right)} d \theta} d \theta .
\end{aligned}
$$

Following the same steps mentioned above, we find

$h(\theta)=e^{-a \mu(\Theta)}, L(\theta, x)$ and $g(\theta)$ are the same as those given in Eqs. (12) and (19).

$$
\begin{gathered}
\hat{h}_{\theta}=-a \mu^{\prime}(\theta) e^{-a \mu(\theta)}, \hat{h}_{\theta \theta}=-a\left(\mu^{\prime \prime}(\theta)-a\left(\mu^{\prime}(\theta)\right)^{2}\right) e^{-a \mu(\theta)}, \hat{p}_{\theta}=\frac{\beta}{\theta^{2}}-\frac{\alpha+1}{\theta}, \\
\hat{L}_{\theta \theta}=-\frac{3 n}{\theta^{2}}+\frac{n}{(\theta+2)^{2}}, \quad \hat{\sigma}_{\theta \theta}=\frac{\theta^{2}(\theta+2)^{2}}{n\left[3(\theta+2)^{2}+\theta^{2}\right]}, \quad \hat{L}_{\theta \theta \theta}=\frac{6 n}{\theta^{3}}-\frac{2 n}{(\theta+2)^{3}},
\end{gathered}
$$

Then, we get

$$
\mathbf{P}_{\mathrm{LIN}}^{B}=-\frac{1}{a} \ln \left[\begin{array}{c}
e^{-a \mu(\hat{\theta})}+0.5\left[\left(\begin{array}{c}
\left(-a\left(\mu^{\prime}(\hat{\theta})-a\left(\mu^{\prime}(\hat{\theta})\right)^{2}\right) e^{-a \mu(\hat{\theta})}\right)+ \\
2\left(-a \mu^{\prime}(\hat{\theta}) e^{-a \mu(\hat{\theta})}\right)\left(\frac{\beta}{\theta^{2}}-\frac{\alpha+1}{\theta}\right)
\end{array}\right] \frac{\hat{\theta}^{2}\left(\hat{\theta}^{2}+2\right)}{n\left[3\left(\hat{\theta}^{2}+2\right)^{2}+2 \hat{\theta}^{2}\left(2-\hat{\theta}^{2}\right)\right]}\right] \\
+0.5\left[\left(\frac{\hat{\theta}^{2}\left(\hat{\theta}^{2}+2\right)}{n\left[3\left(\hat{\theta}^{2}+2\right)^{2}+2 \hat{\theta}^{2}\left(2-\hat{\theta}^{2}\right)\right]}\right)^{2}\left(-a \mu^{\prime}(\hat{\theta}) e^{-a \mu(\hat{\theta})}\right)\left(\frac{6 n}{\hat{\theta}^{3}}-\frac{4 n\left(\hat{\theta}^{3}-6 \hat{\theta}\right)}{\left(\hat{\theta}^{2}+2\right)^{3}}\right)\right]
\end{array}\right]
$$

\subsection{Bayesian Premium Estimators Under Entropy Loss Function}

\subsubsection{Posterior distribution using the extension of Jeffreys prior}

Using the entropy loss function, the corresponding Bayesian premium estimator is as follows

$$
\begin{gathered}
\mathbf{P}_{\mathrm{ENT}}^{B}=\left(E\left[\mu(\Theta)^{-1} \mid x\right]\right)^{-1} . \\
E\left[\mu(\Theta)^{-1} \mid x\right]=\int_{0}^{\infty} \mu(\theta)^{-1} f(\theta \mid x) d \theta \\
=\frac{\int_{0}^{\infty} \frac{\theta^{(3 n-2 c)}}{(2 \theta+6)(\theta+2)^{n+2 c-1}}\left(\theta^{2}+6 \theta+6\right)^{c} e^{-\theta \sum_{i=1}^{n} x_{i}}}{\int_{0}^{\infty} \frac{\theta^{(3 n-2 c)}}{(2 \theta+6)(\theta+2)^{n+2 c-1}}\left(\theta^{2}+6 \theta+6\right)^{c} e^{-\theta \sum_{i=1}^{n} x_{i}} d \theta} \\
=\frac{\int_{\theta} h(\theta) \exp [L(\theta, x)+g(\theta)] d \theta}{\int_{\theta} \exp [L(\theta, x)+g(\theta)] d \theta}, \quad \theta>0 \\
h(\theta)=\mu(\theta)^{-1}=\frac{1}{\mu(\theta)}=\frac{\theta(\theta+2)}{2(\theta+3)},
\end{gathered}
$$

$L(\theta, x)$ and $g(\theta)$ are the same as those given in Eqs. (12) and (13)

$$
\begin{gathered}
\hat{h}_{\theta}=\frac{2 \theta^{2}+12 \theta+12}{(2 \theta+6)^{2}}, \quad \hat{h}_{\theta \theta}=\frac{24}{(2 \theta+6)^{2}}, \quad \hat{p}_{\theta}=2 c\left(\frac{\theta+3}{\theta^{2}+6 \theta+6}-\frac{2 \theta+2}{\theta^{2}+2 \theta}\right), \\
\hat{L}_{\theta \theta}=-\frac{3 n}{\theta^{2}}+\frac{n}{(\theta+2)^{2}}, \hat{\sigma}_{\theta \theta}=\frac{\theta^{2}(\theta+2)^{2}}{n\left[3(\theta+2)^{2}+\theta^{2}\right]}, \hat{L}_{\theta \theta \theta}=\frac{6 n}{\theta^{3}}-\frac{2 n}{(\theta+2)^{3}},
\end{gathered}
$$


Then, we get

$$
\left.\begin{array}{c}
\mathbf{P}_{\mathrm{LIN}}^{B}=E\left[e^{-a \mu(\Theta)} \mid x\right] \\
{\left[\begin{array}{c}
\frac{\hat{\theta}(\hat{\theta}+2)}{\hat{\theta}^{2}+2 \hat{\theta}}+0.5\left[\left(\frac{-8 \theta^{3}+144 \theta}{\left(\theta^{2}+6\right)^{3}}\right)+\right. \\
4 c\left(\frac{\theta^{4}+16 \theta^{2}+12}{\left(\theta^{2}+6\right)^{2}}\right)\left(\frac{2 \theta^{3}+16 \theta}{\theta^{4}+16 \theta+12}-\frac{3 \theta^{2}+2}{\theta^{3}+2 \theta}\right) \\
0.5\left[\left(\frac{\hat{\theta}^{2}\left(\hat{\theta}^{2}+2\right)}{n\left[3\left(\hat{\theta}^{2}+2\right)^{2}+2 \hat{\theta}^{2}\left(2-\hat{\theta}^{2}\right)\right]}\right)^{2}\left(\frac{\left.\hat{\theta}^{2}+16 \hat{\theta}^{2}+2\right)}{\left(\hat{\theta}^{2}+6\right)^{2}}\right)\left(\frac{6 n}{\hat{\theta}^{3}}-\frac{4 n\left(\hat{\theta}^{2}-6 \hat{\theta}\right)}{\left(\hat{\theta}^{2}+2\right)^{3}}\right)\right]
\end{array}\right]}
\end{array}\right] .
$$

\subsubsection{Posterior distribution using the IG prior}

The corresponding Bayes estimator for the parameter $\theta$ under the entropy loss function is:

$$
\begin{gathered}
\mathbf{P}_{\mathrm{Ent}}^{B}=E\left[\mu(\Theta)^{-1} \mid x\right]^{-1} . \\
E\left[\mu(\Theta)^{-1} \mid x\right]=\int_{0}^{\infty} \mu(\theta)^{-1} f(\theta \mid x) d \theta=\frac{\int_{0}^{\infty} \frac{\theta^{3 n-\alpha}}{2(2+\theta)^{n-1}(\theta+3)} e^{-\frac{\beta}{\theta}-\theta \sum_{i=1}^{n} x_{i}} d \theta}{\int_{0}^{\infty} \frac{\theta^{3 n-(\alpha+1)}}{(2+\theta)^{n}} e^{-\frac{\beta}{\theta}-\theta \sum_{i=1}^{n} x_{i}} d \theta} .
\end{gathered}
$$

Following the same steps mentioned above, we find

$h(\theta)=\frac{\theta(\theta+2)}{(2 \theta+6)}, L(\theta, x)$ and $g(\theta)$ are the same as those given in Eqs. (12) and (19).

$$
\begin{gathered}
\hat{h}_{\theta}=\frac{2 \theta^{2}+12 \theta+12}{(2 \theta+6)^{2}}, \quad \hat{h}_{\theta \theta}=\frac{24}{(2 \theta+6)^{2}}, \quad \hat{p}_{\theta}=\frac{\beta}{\theta^{2}}-\frac{\alpha+1}{\theta}, \\
\hat{L}_{\theta \theta}=-\frac{3 n}{\theta^{2}}+\frac{n}{(\theta+2)^{2}}, \hat{\sigma}_{\theta \theta}=\frac{\theta^{2}(\theta+2)^{2}}{n\left[3(\theta+2)^{2}+\theta^{2}\right]}, \hat{L}_{\theta \theta \theta}=\frac{6 n}{\theta^{3}}-\frac{2 n}{(\theta+2)^{3}},
\end{gathered}
$$

Then, we get

$$
\begin{aligned}
& \mathbf{P}_{\mathrm{ENT}}^{B}=E\left[e^{-a \mu(\Theta)} \mid x\right] \\
& =\left[\begin{array}{c}
\frac{\hat{\theta}(\hat{\theta}+2)}{2 \hat{\theta}+6}+0.5\left[\left(\frac{\left(\frac{-8 \theta^{3}+144 \theta}{\left(\theta^{2}+6\right)^{3}}\right)+}{2\left(\frac{\theta^{4}+16 \theta^{2}+12}{\left(\theta^{2}+6\right)^{2}}\right)\left(\frac{\beta}{\theta^{2}}-\frac{\alpha+1}{\theta}\right)}\right) \frac{\hat{\theta}^{2}\left(\hat{\theta}^{2}+2\right)}{n\left[3\left(\hat{\theta}^{2}+2\right)^{2}+2 \hat{\theta}^{2}\left(2-\hat{\theta}^{2}\right)\right]}\right]+ \\
0.5\left[\left(\frac{\hat{\theta}^{2}\left(\hat{\theta}^{2}+2\right)}{n\left[3\left(\hat{\theta}^{2}+2\right)^{2}+2 \hat{\theta}^{2}\left(2-\hat{\theta}^{2}\right)\right]}\right)^{2}\left(\frac{\hat{\theta}^{4}+16 \hat{\theta}^{2}+12}{\left(\hat{\theta}^{2}+6\right)^{2}}\right)\left(\frac{6 n}{\hat{\theta}^{3}}-\frac{4 n\left(\hat{\theta}^{3}-6 \hat{\theta}\right)}{\left.\left(\hat{\theta}^{2}+2\right)^{3}\right)}\right]\right.
\end{array}\right] .
\end{aligned}
$$

\subsubsection{Elicitation of hyper-parameter(s)}

According to [28], elicitation is the process of formulating a person's knowledge and beliefs about one or more uncertain quantities into a (joint) probability distribution for those quantities. In the context of Bayesian statistical analysis, it arises most usually as a method for specifying the prior distribution for one or more unknown parameters of a statistical model. It is a difficult task because we first have to identify the prior distribution and then its hyper-parameters.

In this article, we focus on the method proposed by [29] to determine the hyper-parameters $\alpha$ and $\beta$ of the gamma prior, this method is based on bootstrap method, we adopt the same steps explained in [14]. 


\section{SIMULATION STUDY}

In this section, Monte Carlo simulation study is performed to compare the methods of estimation by using mean square Errors (MSE's) as follows:

$$
\operatorname{MSE}\left(\hat{P}^{B}\right)=\frac{\sum_{i=1}^{N}\left(\hat{P}^{B}-\mu(\theta)\right)^{2}}{N} .
$$

Where $N$ is the number of replications. We generated 100,000 samples of size $n=20,40,60,80,100,1,000$ and 10,000 to represent small, moderate and large sample sizes from ZD with three values of $(\theta=0.44,1.5,9)$. In order to compare the Bayesian premium estimators obtained in the above section under three different loss functions and two priors, we choose the values of the extensive Jeffreys constants, $(c=0.01,0.5,1)$ and for the IG prior, the following pairs of values of the hyper parameters $\alpha$ and $\beta$ are chosen $(\alpha, \beta)=(0.2,0.3),(1,1.5)$ with two values of linex loss symmetry $(a= \pm 0.01)$ and $q=1$ for entropy loss (Figure 1$)$.
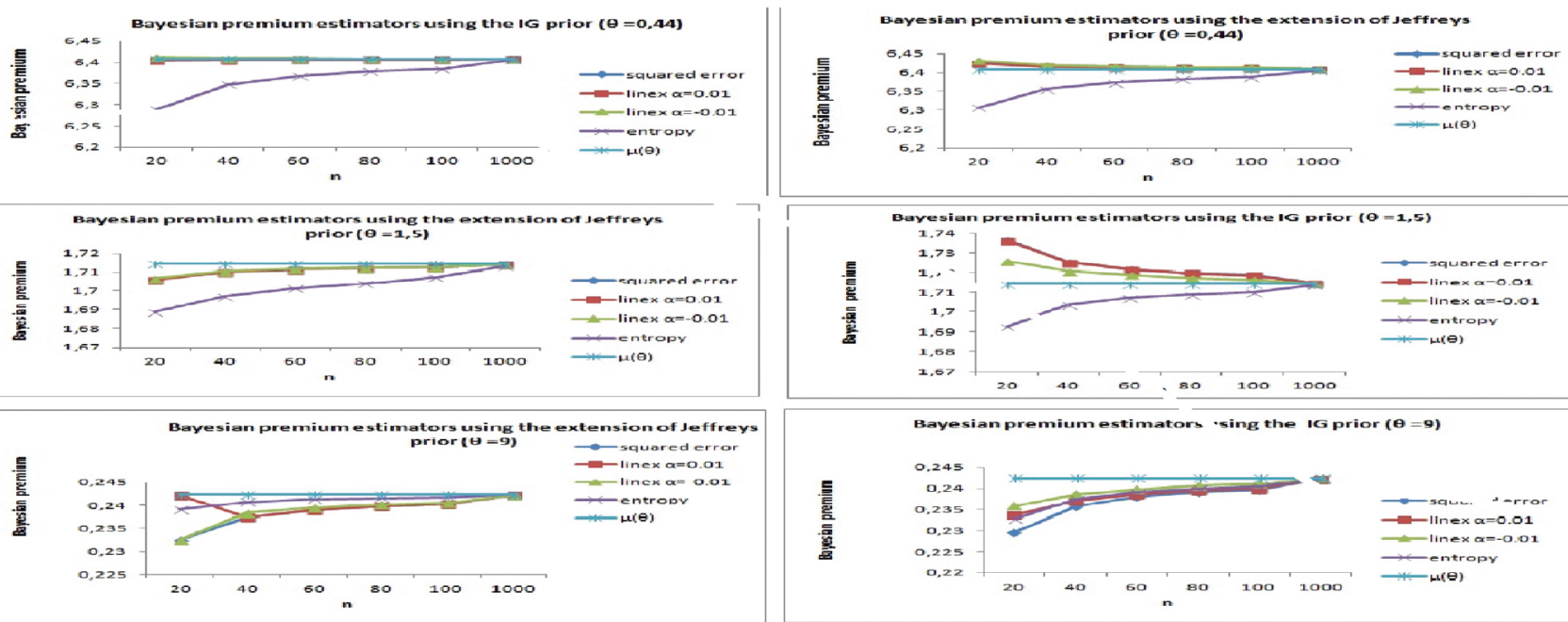

Figure 1 Bayesian premium estimators using different methods and values.

The results are summarized and tabulated in the following Tables 1-4.

Table 1 Bayesian premium estimators and respective MSE's under squared error loss function $(\alpha=0.2, \beta=0.3, c=0.01)$.

\begin{tabular}{llll}
\hline$\theta$ & 0.44 & 1.5 & 9.0 \\
\hline$\mu(\theta)$ & 6.408346 & 1.714286 & 0.2424242 \\
\hline$n$ & & Ext.J.P & \\
\hline 20 & 6.427768 & 1.70642 & 0.2325904 \\
& $(0.0003772195)$ & $(6.186881 e-05)$ & $(9.670397 e-05)$ \\
\hline 40 & 6.418092 & 1.710361 & 0.2374164 \\
& $(9.499057 e-05)$ & $(1.540085 e-05)$ & $(2.507806 e-05)$ \\
\hline 60 & 6.414851 & 1.711671 & 0.239065 \\
& $(4.232033 e-05)$ & $(6.835437 e-06)$ & $(1.128418 e-05)$ \\
\hline 80 & 6.413228 & 1.712326 & 0.239897 \\
& $(2.383403 e-05)$ & $(3.842341 e-06)$ & $(6.386787 e-06)$ \\
\hline 100 & 6.412253 & 1.712718 & 0.2403987 \\
& $(1.526487 e-05)$ & $(2.458112 e-06)$ & $(4.102794 e-06)$ \\
\hline 1000 & 6.408737 & 1.714129 & 0.2422203 \\
& $(1.53049 e-07)$ & $(2.454623 e-08)$ & $(4.15837 e-08)$ \\
\hline & & & $($ continued $)$
\end{tabular}


Table 1 Bayesian premium estimators and respective MSE's under squared error loss function $(\alpha=0.2, \beta=0.3, c=0.01)$. (Continued)

\begin{tabular}{llll}
\hline$\theta$ & 0.44 & 1.5 & 9.0 \\
\hline$\mu(\theta)$ & 6.408346 & 1.714286 & 0.2424242 \\
\hline$n$ & & IG.P & \\
\hline 20 & 6.408436 & 1.73643 & 0.2296947 \\
& $(8.184013 e-09)$ & $(0.0004903582)$ & $(0.0001620411)$ \\
\hline 40 & 6.408226 & 1.725384 & 0.2359006 \\
& $(1.427129 e-08)$ & $(0.0001231621)$ & $(4.255847 e-05)$ \\
\hline 60 & 6.408229 & 1.72169 & 0.2380387 \\
& $(1.361046 e-08)$ & $(5.482373 e-05)$ & $(1.923311 e-05)$ \\
\hline 80 & 6.408244 & 1.719841 & 0.2391212 \\
& $(1.029034 e-08)$ & $(3.086227 e-05)$ & $(1.090976 e-05)$ \\
\hline 100 & 6.408258 & 1.718731 & 0.2397752 \\
& $(7.719616 e-09)$ & $(1.976105 e-05)$ & $(7.017584 e-06)$ \\
\hline 1000 & 6.408335 & 1.714731 & 0.2421569 \\
& $(1.256809 e-10)$ & $(1.979417 e-07)$ & $(7.14696 e-08)$ \\
\hline
\end{tabular}

Table 2 Bayesian premium estimators and respective MSE's under linex loss function $(\alpha=0.2, \beta=0.3, a=0.01, c=0.5)$.

\begin{tabular}{|c|c|c|c|}
\hline$\theta$ & 0.44 & 1.5 & 9.0 \\
\hline$\mu(\theta)$ & 6.408346 & 1.714286 & 0.2424242 \\
\hline$n$ & & Ext.J.P & \\
\hline \multirow[t]{2}{*}{20} & 6.423919 & 1.706052 & 0.2325945 \\
\hline & $(0.0002425291)$ & $(6.779723 e-05)$ & $(9.662431 e-05)$ \\
\hline \multirow[t]{2}{*}{40} & 6.416157 & 1.710176 & 0.2374184 \\
\hline & $(6.100882 e-05)$ & $(1.689136 e-05)$ & $(2.505827 e-05)$ \\
\hline \multirow[t]{2}{*}{60} & 6.413558 & 1.711547 & 0.2390664 \\
\hline & $(2.717113 e-05)$ & $(7.499168 e-06)$ & $(1.127541 e-05)$ \\
\hline \multirow[t]{2}{*}{80} & 6.412257 & 1.712232 & 0.239898 \\
\hline & $(1.529957 e-05)$ & $(4.216053 e-06)$ & $(6.381861 e-06)$ \\
\hline \multirow[t]{2}{*}{100} & 6.411476 & 1.712643 & 0.2403995 \\
\hline & $(9.797806 e-06)$ & $(2.697428 e-06)$ & $(4.099645 e-06)$ \\
\hline \multirow[t]{2}{*}{1000} & 6.408659 & 1.714122 & 0.2422204 \\
\hline & $(9.819732 e-08)$ & $(2.694448 e-08)$ & $(4.155233 e-08)$ \\
\hline$n$ & & IG.P & \\
\hline \multirow[t]{2}{*}{20} & 6.404592 & 1.736061 & 0.2338005 \\
\hline & $(1.408841 e-05)$ & $(0.0004741641)$ & $(7.436952 e-05)$ \\
\hline \multirow[t]{2}{*}{40} & 6.406292 & 1.725198 & 0.2371807 \\
\hline & $(4.217769 e-06)$ & $(0.000119076)$ & $(2.749423 e-05)$ \\
\hline \multirow[t]{2}{*}{60} & 6.406937 & 1.721566 & 0.2386573 \\
\hline & $(1.985068 e-06)$ & $(5.300208 e-05)$ & $(1.418976 e-05)$ \\
\hline \multirow[t]{2}{*}{80} & 6.407274 & 1.719748 & 0.239485 \\
\hline & $(1.148483 e-06)$ & $(2.983602 e-05)$ & $(8.639111 e-06)$ \\
\hline \multirow[t]{2}{*}{100} & 6.407481 & 1.718656 & 0.2400145 \\
\hline & $(7.47438 e-07)$ & $(1.910364 e-05)$ & $(5.806893 e-06)$ \\
\hline \multirow[t]{2}{*}{1000} & 6.408257 & 1.714723 & 0.2421813 \\
\hline & $(7.9315 e-09)$ & $(1.913457 e-07)$ & $(5.904271 e-08)$ \\
\hline
\end{tabular}


Table 3 Bayesian premium estimators and respective MSE's under $(\alpha=0.2, \beta=0.3, a=-0.01, c=0.5)$.

\begin{tabular}{|c|c|c|c|}
\hline$\theta$ & 0.44 & 1.5 & 9.0 \\
\hline$\mu(\theta, \gamma)$ & 6.408346 & 1.714286 & 0.2424242 \\
\hline$n$ & & Ext.J.P & \\
\hline \multirow[t]{2}{*}{20} & 6.431615 & 1.706788 & 0.2325864 \\
\hline & $(0.0005414665)$ & $(5.621073 e-05)$ & $(9.678369 e-05)$ \\
\hline \multirow[t]{2}{*}{$\overline{40}$} & 6.420027 & 1.710547 & 0.2384016 \\
\hline & $(0.0001364558)$ & $(1.397906 e-05)$ & $(1.618175 e-05)$ \\
\hline \multirow[t]{2}{*}{60} & 6.416144 & 1.711795 & 0.2395387 \\
\hline & $(6.080963 e-05)$ & $(6.202423 e-06)$ & $(8.326238 e-06)$ \\
\hline \multirow[t]{2}{*}{80} & 6.414198 & 1.712419 & 0.2401746 \\
\hline & $(3.425128 e-05)$ & $(3.485955 e-06)$ & $(5.06069 e-06)$ \\
\hline \multirow[t]{2}{*}{100} & 6.41303 & 1.712792 & 0.2405809 \\
\hline & $(2.193846 e-05)$ & $(2.229904 e-06)$ & $(3.397942 e-06)$ \\
\hline \multirow[t]{2}{*}{1000} & 6.408815 & 1.714137 & 0.2422388 \\
\hline & $(2.200214 e-07)$ & $(2.225972 e-08)$ & $(3.439732 e-08)$ \\
\hline$n$ & & IG.P & \\
\hline \multirow[t]{2}{*}{20} & 6.41228 & 1.725569 & 0.2358985 \\
\hline & $(1.547928 e-05)$ & $(0.0001273162)$ & $(4.258488 e-05)$ \\
\hline \multirow[t]{2}{*}{40} & 6.410161 & 1.72074 & 0.238655 \\
\hline & $(3.293498 e-06)$ & $(4.165851 e-05)$ & $(1.420682 e-05)$ \\
\hline \multirow[t]{2}{*}{60} & 6.409521 & 1.718806 & 0.2397744 \\
\hline & $(1.382034 e-06)$ & $(2.042952 e-05)$ & $(7.021747 e-06)$ \\
\hline \multirow[t]{2}{*}{80} & 6.409215 & 1.7173 & 0.2406517 \\
\hline & $(7.548 e-07)$ & $(9.085614 e-06)$ & $(3.141889 e-06)$ \\
\hline \multirow[t]{2}{*}{100} & 6.409035 & 1.716547 & 0.2410926 \\
\hline & $(4.744779 e-07)$ & $(5.112298 e-06)$ & $(1.773294 e-06)$ \\
\hline \multirow[t]{2}{*}{1000} & 6.408412 & 1.714512 & 0.2422905 \\
\hline & $(4.440555 e-09)$ & $(5.116727 e-08)$ & $(1.789591 e-08)$ \\
\hline
\end{tabular}

Table 4 Bayesian premium estimators and respective MSE's under entropy loss function $(\alpha=1, \beta=1.5, q=1, c=1)$.

\begin{tabular}{llll}
\hline$\theta$ & $\mathbf{0 . 4 4}$ & $\mathbf{1 . 5}$ & $\mathbf{9}$ \\
\hline$\mu(\theta)$ & 6.408346 & 1.714286 & 0.2424242 \\
\hline$n$ & & $\mathbf{E x t . J . P}$ & 0.2390914 \\
\hline 20 & 6.307508 & 1.688745 & $(1.110798 e-05)$ \\
\hline 40 & $(0.01016832)$ & $(0.0006523198)$ & 0.2407382 \\
& 6.357651 & 1.697218 & $(2.842876 e-06)$ \\
\hline 60 & $(0.002569982)$ & $(0.0002913227)$ & 0.2412957 \\
& 6.374487 & 1.701469 & $(1.273515 e-06)$ \\
\hline 80 & $(0.001146389)$ & $(0.0001642655)$ & 0.2415762 \\
& 6.382929 & 1.704025 & $(7.191958 e-07)$ \\
\hline 100 & $(0.0006460224)$ & $(0.0001052825)$ & 0.241745 \\
& 6.388001 & 1.707439 & $(4.61383 e-07)$ \\
\hline 1000 & $(0.0004139078)$ & $(4.688288 e-05)$ & 0.242356 \\
& 6.406307 & 1.713257 & $(4.653705 e-09)$ \\
\hline & $(4.155459 e-06)$ & $(1.058344 e-06)$ & $($ continued $)$
\end{tabular}


Table 4 Bayesian premium estimators and respective MSE's under entropy loss function

\begin{tabular}{llll}
$(\alpha=1, \beta=1.5, q=1, c=1) .($ Continued) & & \\
\hline$\theta$ & $\mathbf{0 . 4 4}$ & $\mathbf{1 . 5}$ & $\mathbf{9}$ \\
\hline$\mu(\theta)$ & 6.408346 & 1.714286 & 0.2424242 \\
\hline$n$ & & IG.P & \\
\hline 20 & 6.28851 & 1.693029 & 0.2327684 \\
& $(0.01651358)$ & $(0.1591305)$ & $(0.01352628)$ \\
\hline 40 & 6.347871 & 1.703629 & 0.237462 \\
& $(0.004780887)$ & $(0.1676992)$ & $(0.01464006)$ \\
\hline 60 & 6.367904 & 1.707175 & 0.2390849 \\
& $(0.002411886)$ & $(0.170616)$ & $(0.01503543)$ \\
\hline 80 & 6.377967 & 1.70895 & 0.2399079 \\
& $(0.001524722)$ & $(0.1720858)$ & $(0.01523793)$ \\
\hline 100 & 6.38402 & 1.710016 & 0.2404054 \\
& $(0.001088649)$ & $(0.1729713)$ & $(0.01536101)$ \\
\hline 1000 & 6.405905 & 1.713858 & 0.2422203 \\
& $(0.0001234279)$ & $(0.1761822)$ & $(0.01581417)$ \\
\hline
\end{tabular}

\section{DISCUSSION}

This study deals with the Bayesian estimation problem based on ZD as a conditional distribution. For Bayesian premium estimators, the performance depends on the form of the prior distribution, and the loss function assumed. Most authors used squared error as a symmetric loss function. However, in practice, the real loss function is often not symmetric. The simulation study revealed that the Bayesian premium estimator under entropy loss is also more efficient than the Bayes estimator under squared error and Linex loss functions in most of the situation. Furthermore, MSE of the Bayesian premium estimators for the entropy loss has the smallest values as compared with the corresponding Bayesian estimators under Linex and squared error loss functions. It may be noted here that when $\theta$ increases, $\mu(\theta)$ decreases and the Bayesian premium estimator tends to $\mu(\theta)$. Under the two above priors, we conclude that the performance is approximately equal to smaller posterior risk as compared. Also, the results of the gamma prior are more precise than an extension of Jeffreys prior. From the above mentioned discussion, we may conclude that the Bayes procedure discussed in this paper can be recommended for their use.

\section{CONCLUSION}

In this paper, since the risk parameter for a policyholder is never known, we constructed Bayesian premium estimators following Bayesian inference techniques. By imposing a prior distribution on, we are able to probabilistically describe the risk structure for the entire rating class. In practice, the choice of this prior distribution is subjective to personal judgments or induced from historical data of the corresponding group. Using numerical simulation, it seems that the Bayesian premiums are consistent and verified the condition of convergence to the individual premium. For future studies, we can consider the distributions of inverse Lindley, gamma-Lindley as a conditional distribution instead of ZD, under entropy, linex and squared error loss functions respectively. In addition, this work can be extended using censored data.

\section{ACKNOWLEDGMENTS}

The authors acknowledge Editor in chief, Prof. Mohammad Ahsanullah and the referee, of this journal for the constant encouragement to finalize the paper. Their comments and suggestions greatly improved the article.

\section{REFERENCES}

1. A.L. Bailey, Proc. Casual. Actuar. Soc. 37 (1950), 7-23.

2. H. Zeghdoudi, H. Messaadia, Int. J. Comput. Sci. Math. 9(1) (2018), 58-65.

3. M.E. Ghitany, B. Atieh, S. Nadarajah, Math.Comput. Simulat. 78(2008), 493-506.

4. D.V. Lindley, J. R. Stat. Soc. Ser. A. 20 (1958). 102-107.

5. H. Zeghdoudi, S. Nedjar, J. Appl. Probab. Stat. 11. 1. (2016), 129-138.

6. H. Zeghdoudi, S. Nedjar. J. Afr. Stat. 11. 1. (2016), 923-932.

7. H. Zeghdoudi, S. Nedjar. J. Comput. Appl. Math. 298. (2016), 167-174.

8. H. Zeghdoudi, S. Nedjar. J. New Trends Math. Sci. 5. 1. (2017), 59-65. 
9. H. Zeghdoudi, N. Lazri, JGSTF JMSOR. 3(2) (2016), 1-7

10. R. Shanker, S. Sharma, R. Shanker. Appl. Math. 42 (2013), 1-6

11. A. Zellner. J. Ams. Stat. Assoc. 81. (1986), 446-451.

12. M. Sankaran, Biometrics.26 (1970), 145-149.

13. M.E. Ghitany, D.K. Al-Mutairi. J. Stat. Comput. Simul. 79. 1. (2009), 1-9.

14. H. Krishna, K. Kumar.Math. Comput. Simul.82(2) (2011), 281-294.

15. S. Ali, M. Aslam, S.M.A. Kazmi, Appl. Math. Model. 37 (2013), 6068-6078.

16. F. Metiri, H. Zeghdoudi, M.R. Remita. Global J Pure Appl Math. 12 (1) (2016) 391-400.

17. A. Legendre, Nouvelles méthodes pour la determination des orbites des comètes, Courcier, City Paris, 1805.

18. H.S. Al-Kutubi, N.A. Ibrahim. Malay. J. Math. Sci. 32 (2009), 297-313.

19. D.V. Lindley, Trab. Invest. Oper.31(1) (1980), 223-245.

20. H.A. Howlader, A. Hossain, Comput. Stat. Data. Anal.38 (2002), 301-314.

21. Z.F. Jaheen, J. Stat.Comput. Simul.75(2005), 1-11.

22. Rojo. Commun. Stat. Theory Methods. 116. (1987) 3745-3748.

23. A.P. Basu, Ebrahimi N. J. Stat. Plann. Infer. 29. (1991) 21-31.

24. B.N. Pandey, Commun. Stat. Theory Methods.26(9) (1997), 2191-2202.

25. R.D. Thompson, A.P. Basu, J. Bayesian Anal. Stat. Econ. Wiley, New York, 1996.

26. M. Nassar, F.H. Eissa. Commun. Stat. Theory Methods. 33. 10. (2005) 2343-2362.

27. R. Calabria, G. Pulcini,Commun. Stat. Theory Methods.25(3) (1969), 585-600.

28. H.P. Garthwaite, B.J. Kadane, A. O'Hagan. Elicitation, working paper in university of Sheffield, 2004.

29. S.E. Ahn, C.S. Park, H.M. Kim, Stoch. Environ. Res. Assess.21(2007), 711-716. 